\title{
Approaches toward optimized laser- induced damage thresholds of dispersive compensating mirrors applying nanolaminates
}

T. Willemsen, M. Brinkmann, M. Jupé, M. Gyamfi, S. Schlichting, et al.

T. Willemsen, M. Brinkmann, M. Jupé, M. Gyamfi, S. Schlichting, D. Ristau, "Approaches toward optimized laser-induced damage thresholds of dispersive compensating mirrors applying nanolaminates," Proc. SPIE 10447, Laser-Induced Damage in Optical Materials 2017, 1044712 (22 March 2018); doi: $10.1117 / 12.2281126$

SPIE. Event: SPIE Laser Damage, 2017, Boulder, Colorado, United States 


\title{
Approaches toward optimized laser-induced damage thresholds of dispersive compensating mirrors applying nanolaminates
}

\author{
T. Willemsen, ${ }^{a, b},{ }^{*}$ M. Brinkmann, ${ }^{b}$ M. Jupé, ${ }^{b}$ M. Gyamfi, ${ }^{b}$ S. Schlichting and D. Ristau ${ }^{\mathrm{a}, \mathrm{b}}$ \\ ${ }^{a}$ Institut für Quantenoptik Quest, Leibniz University, Hanover, 30167, Germany \\ ${ }^{b}$ Laser Zentrum Hannover e.V., Hollerithallee 8, 30419, Hanover, Germany
}

\begin{abstract}
Ultra-short laser applications require high quality dielectric optics. The natural dispersion of light needs to be matched by dielectric components. However such dispersive components are very challenging for the deposition process and are characterized by high field intensities inside the layer stack. Such layers are expected to diminish the possible laser induced damage thresholds (LIDTs) because of their low optical gap value for suitable high refractive index materials. This paper reports about the manufacturing of amorphous nanolaminates to tune the optical gap. Such sequences are substituted into a conventional high reflective mirror to decrease the electric field of binary Tantala layers by $30 \%$ which correlates to an improvement in LIDT of almost $16 \%$.
\end{abstract}

Keywords: Quantum film, nanolaminate, LIDT measurements, new material properties

\section{Introduction}

Laser applications in the femtosecond (fs) regime are required for a huge quantity of direct industrial applications and can be considered as a key tool for many basic research projects. In general two laser parameters are essential to fulfill upcoming challenges; the laser pulse duration and the laser peak power. The generation of ultra-short laser pulses in the few fs regime requests optical switches for mode locking. A well-established principle is based on the Kerr effect: The index of refraction is influenced for high laser intensities by an additive term. Highest intensity laser modes are focused stronger in a medium with respect to low intensity modes. The mode locking is realized by adjusting an aperture behind the Kerr media in order to block the low intensity laser modes[1, 2]. However the impact of the natural dispersion of light contributes significantly for such short pulses, leading to time broadened laser pulses. A precise management of the phase of the electric light is necessary to counter the dispersion. One approach can be realized with prism pairs to chirp the laser pulse in time [3]. Prism pairs reach their limit for the generation of low fs pulses due to their inherent third order dispersion (TOD) $[4,5]$. This drawback can be overcome with dielectric mirrors capable to compress pulses without the influence of nonlinear-frequency components higher than the group delay dispersion (GDD) [6]. Presently, dielectric chirped mirrors covering several octaves from the visible to the near infra-red spectral (NIR) range are available and enable the generation of laser pulses close to the estimated limit of $3 \mathrm{fs}$ for a Ti:Sapphire laser oscillator [7 - 9]. However the deposition process for fs components is complex. Smallest deviations of the layer thickness with respect to the design target can lead to a total failure of the projected GDD target band. High end deposition processes like Ion-BeamSputtering (IBS) in combination with precise monitoring techniques are required to maintain a stable deposition process [10]. Recently, the monitoring of IBS processes is significantly improved by controlling the group delay dispersion insitu on the moving substrates, applying a fiber based white light interferometer [11]. In addition, the monitoring concept is well suited to control chirped mirrors target specifications in a wavelength range beyond the bandwidth of the in-situ phase monitor $[12,13]$. Besides the optimized monitoring technique, the design of a chirped mirror remains complex with respect to the electric field distribution. Highest field intensities can occur in layers deep inside the layer stack and are often located in the high index materials. Those layers are expected to determine the laser induced damage threshold of the mirror [14]. High refractive index layers with improved optical properties are needed. However, the number of possible binary dielectric materials for high quality coatings is limited. In semiconductor industry the electron confinement of two binary materials can be tuned by embedding a high refractive index layer in a matrix of two low refractive index layers. By choosing the thickness of the high refractive film small enough, the electron confinement can be changed in such structures, which are often addressed as nanolaminates [15]. Recently the formation of nanolaminates was also shown for dielectric amorphous materials $[16,17]$. The optical gap could be tuned accompanied by an improved laser induced damage threshold.

This paper reports about the application of dielectric amorphous nanolaminates in conventional high reflective mirrors in order to increase the damage resistance of the mirrors. First, the potential of amorphous nanolaminates sequences is demonstrated. Afterwards nanolaminate sequences are inserted in a typical high/low stack of an HR mirror for the NIR 
spectral range. Finally, both mirrors are compared with respect to their laser induced damage thresholds which are measured according to ISO 21254 [18]

\section{Principle of dielectric nanolaminates and deposition process}

The electron confinement of a binary high refractive index material $\mathrm{H}$ can be changed if it is embedded in a matrix of two low refractive index layers $\mathrm{L}$. A nanolaminate sequence is defined as a periodic layer structure of $(\mathrm{LH})^{\mathrm{n}} \mathrm{L}$, whereby $n$ defines the number of layer pairs. High refractive index layers are named as quantum wells and low refractive index layers as barriers (Compare depicted principle in fig. 1).
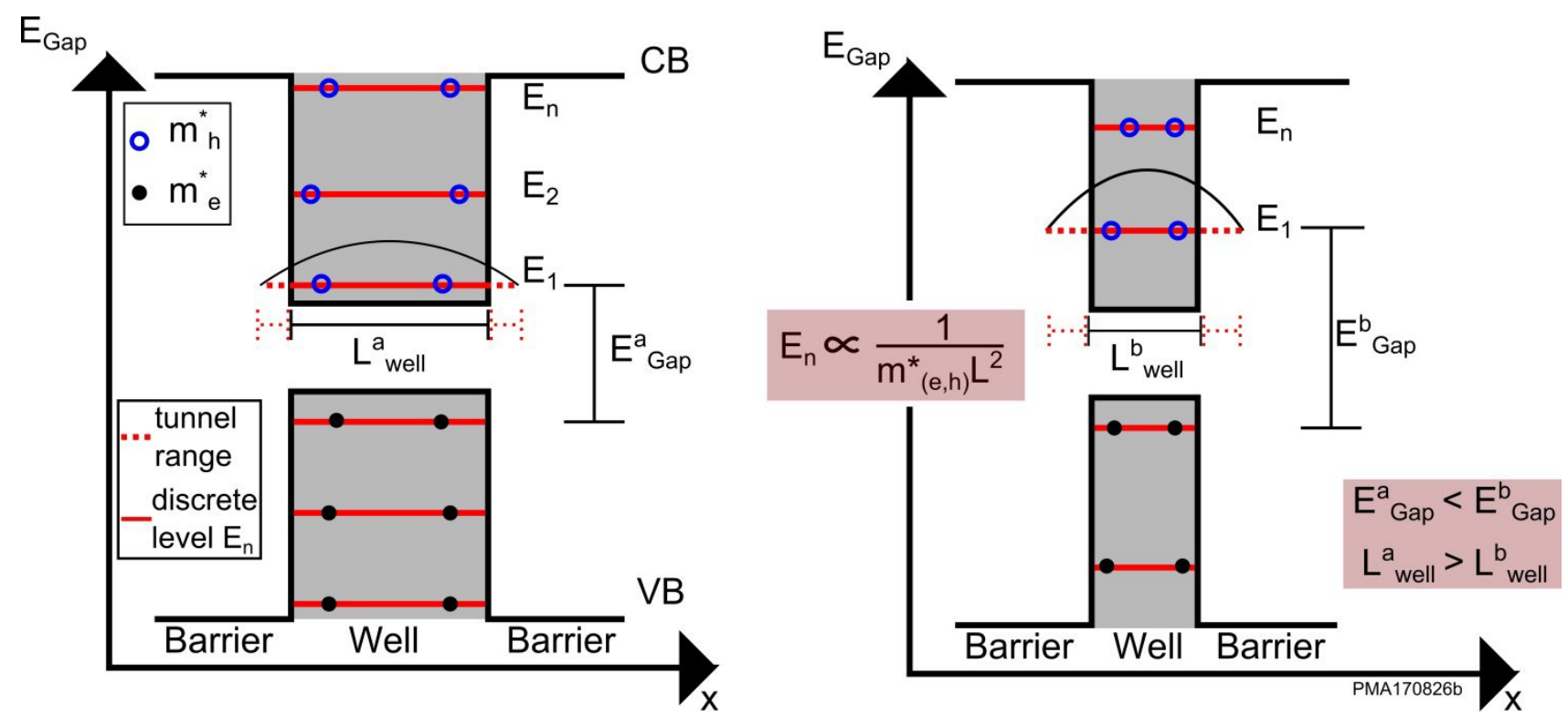

Fig. 1. Schematic illustrating the change of the electron confinement.

The distribution of the energy levels $E_{n}$ in the valance and conduction band can be calculated applying the finite potential well model and numerical solutions of Schrödinger equation $[16,19]$. The energy levels $E_{n}$ are depending on the length of the quantum well $\mathrm{L}_{\text {well }}$ and on the effective mass of the electrons $\mathrm{m}_{\mathrm{e}}^{*}$ and holes $\mathrm{m}_{\mathrm{h}}^{*}$. The electron confinement in terms of the optical gap can be tuned by varying the quantum well. A spectral blue shift occurs for wells of Tantala or Hafnia narrower than $4 \mathrm{~nm}[16,17]$. The electrons can tunnel into specific regions of the barriers approximating an exponential decay $\mathrm{e}^{-\mathrm{ax}}$. As a consequence, the quantum well is broadened by:

$$
\mathrm{L}_{\text {eff }}=\mathrm{L}_{\text {Well }}+2 / \alpha
$$

For instance, the well is effectively broadened by $\mathrm{L}_{\mathrm{eff}}=1.16 \mathrm{~nm}$ assuming an effective mass of the electrons of $\mathrm{m}_{\mathrm{e}}^{*}=0.8$ $\mathrm{m}_{\mathrm{e}}$ for Tantala and a minimum well of $\mathrm{L}_{\mathrm{Well}}=1 \mathrm{~nm}$. The optical gap of nanolaminate sequences applying $1 \mathrm{~nm}$ wells is expected to stay constant for selected barriers thicker than $0.16 \mathrm{~nm}$.

In the following, dielectric nanolaminates of Tantala and Silica are analyzed with varying barrier thicknesses and varying quantum wells, respectively. Every discussed sample is manufactured applying an Ion-Beam-Sputtering process. For sputtering the deposition material from a zone target assembly loaded with Tantalum and Silicon, a $6 \mathrm{~cm}$ Veeco Ion source is operated with argon at a beam current of $150 \mathrm{~mA}$. In reactive gas atmosphere binary Tantala and Silica layers with balanced stoichiometry are deposited and controlled by a precise Broad Band optical Monitoring (BBM) system [10]. The BBM is recording the transmittance every time the rotating monitor substrate is passing the collimator head. In addition the BBM is storing respective transmittance scan within a spectral range from $420 \mathrm{~nm}$ to $1050 \mathrm{~nm}$ after every completed single layer in the coating system. These scans can be automatically loaded to a reoptimization tool, which reviews the dispersion and layer thickness data with respect to the target by evaluating the obtained layer transmittance spectra. In conjunction with a fast refinement algorithm subsequent layers can be optimized [20] (Fig. 2). 


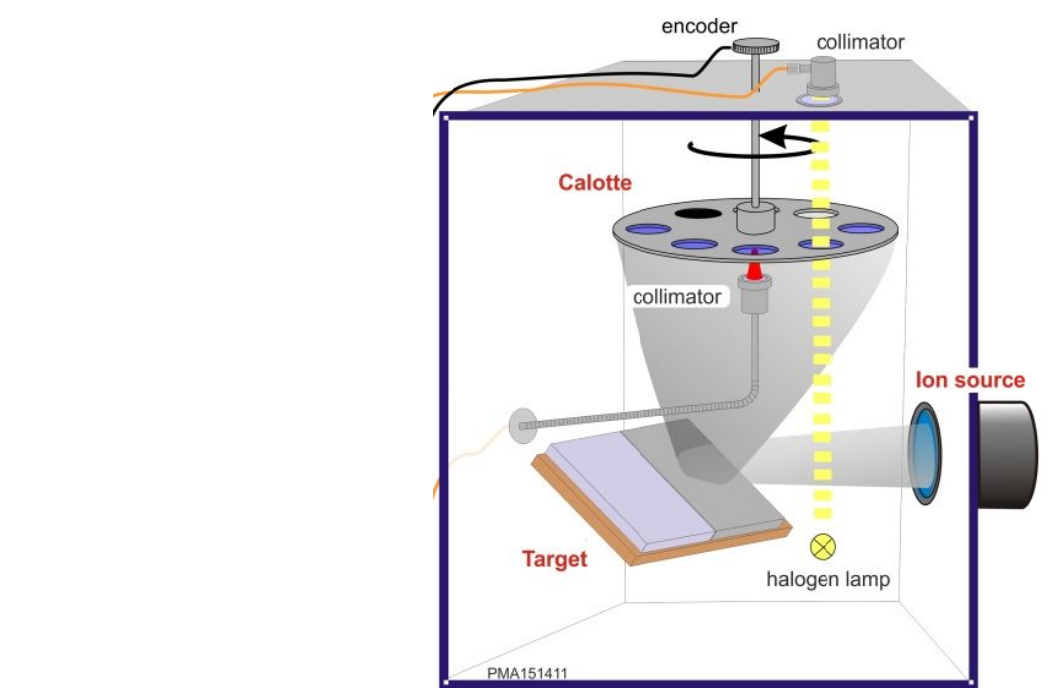

Fig. 2 Principle of the applied IBS process in combination with precise optical monitoring techniques

The periodic structure $(\mathrm{LH})^{\mathrm{n}} \mathrm{L}$ of amorphous nanolaminate stacks is equal to single binary dielectric layers with respect to the spectral behavior. The index of refraction is determined by the Sellmeier formula on the basis of transmittance and reflection measurements [21]. The optical gap is approximated by Tauc procedure [22]. Intermediate states might affect the absolute accuracy for the optical gap, but for a relative comparison of the observed blue shift the Tauc procedure can be considered as a reliable concept [23].

\section{Results of nanolaminate stacks}

The presented working principle of amorphous nanolaminates is only valid for clear steep layer interfaces. A blue shift caused by ternary mixtures of both materials, which may occur at the material interfaces, has to be excluded. A multi-layer stack of $\mathrm{SiO}_{2}$ and $\mathrm{Ta}_{2} \mathrm{O}_{5}$ was manufactured containing different layer thicknesses. The cross-section of this stack system is analyzed applying bright field transmission electron microscopy (Fig. 3 - left). Clear layer interfaces can be identified for sputtered Tantala layers smaller than $2 \mathrm{~nm}$ supporting clearly the formation of a nanolaminate stack (Fig 3. -right).
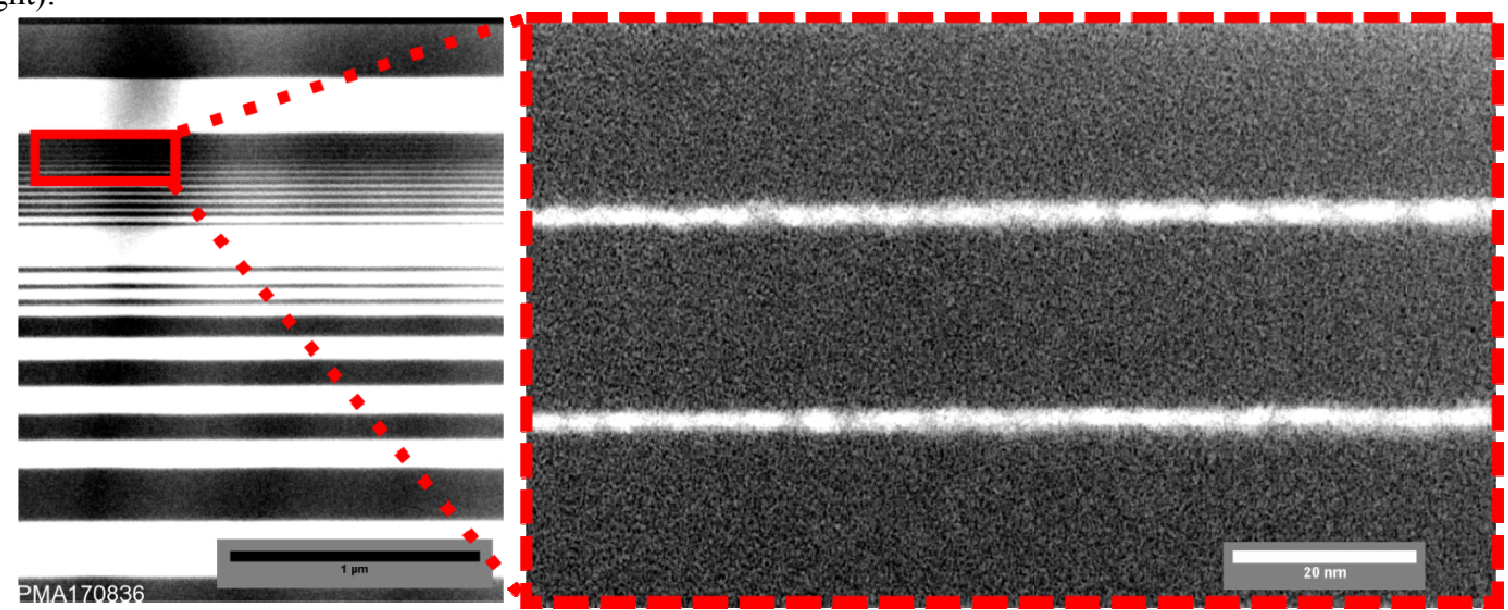

Figure 3. Cross section of a dielectric stack. Dark bars represent Silica layers and brighter bars stand for Tantala layers. The zoomed section depicts good layer interfaces for Tantala layers smaller than $2 \mathrm{~nm}$.

In the following, the influence of varying barrier and well thicknesses and is investigated with respect to the evolution of the optical gap and index of refraction of the nanolaminate sequences. The optical gap is expected to remain constant for a fixed quantum well and a varying barrier above the tunneling range. Five nanolaminate samples were manufactured whereby every sample is characterized by a quantum well of $1 \mathrm{~nm}$. The barrier thickness is varied from 20 , $10,5,1$, and $0.5 \mathrm{~nm}$, respectively. The thickness of the samples is selected to about 3 QWOT (quarter wave optical 
thickness) at a center wavelength of $\lambda_{\mathrm{c}}=800 \mathrm{~nm}$ to ensure a precise calculation of the index of refraction with an accuracy of $\pm 1 \%$. Samples with barriers of $1 \mathrm{~nm}$ include the same total content of $\mathrm{Ta}_{2} \mathrm{O}_{5}$ compared to nanolaminates with $10 \mathrm{~nm}$ in order to exclude blue shift effects induced by decreasing Tantala contents. According to the theoretical prediction, the optical gap remains constant while the index of refraction is increasing exponentially (Fig. 4 - left). Next five nanolaminate samples are manufactured with a varying quantum wells from $8,4,2,1$ and $0.5 \mathrm{~nm}$. In this sample set the barriers are kept constant with $20 \mathrm{~nm}$, respectively. The index of refraction is decreasing linearly with lower wells while the optical gap is increasing exponential in good conjunctions to theoretical models (Fig. 4 - right) [16, 17].
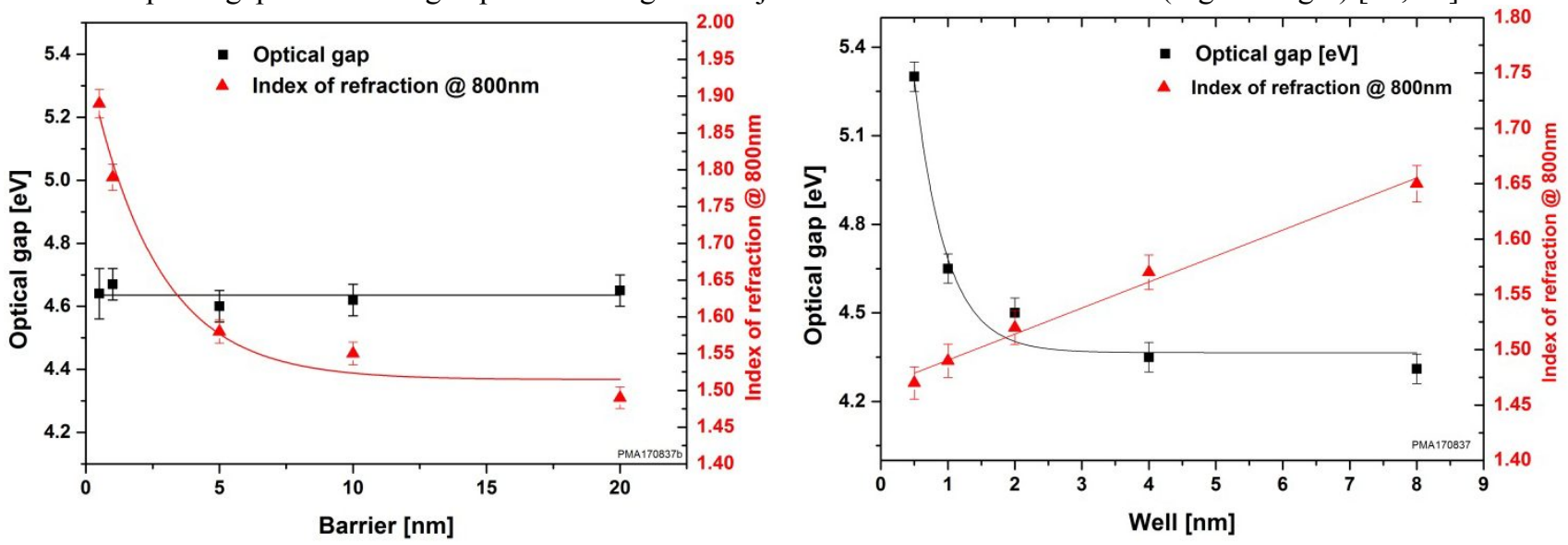

Fig. 4. Evolution of the optical gap and index of refraction for a constant quantum well of $1 \mathrm{~nm}$ (left) and a constant barrier of $20 \mathrm{~nm}$ (right).

\section{Application of nanolaminates in HR-mirrors}

The increasing optical gap is a promising basis to improve the laser induced damage threshold. Following this idea, nanolaminate sequences are integrated in a stack of a conventional high reflective mirror for the wavelength of $800 \mathrm{~nm}$ at an angle of incidence (AOI) at $0^{\circ}$ (Table 1). The design synthesis aims at a significant reduction of the electric field $|\mathrm{E}|$ in binary Tantala layers. The electric field is determined according to the maximum possible value in vacuum, which is the top value in front of an HR-mirror is $100 \%$ and calibrated to an incident wave of $\left|\mathrm{E}_{\text {ind }}\right|=50 \%$. An E-field optimized conventional $(\mathrm{HL})^{15} \mathrm{H}$ design is characterized by a maximum electric field of $47 \%$ in the Tantala layer of the stack next to the surrounding medium (air) (Fig. 5 - left). Afterwards nanolaminate sequences are inserted in the calculated design. An empirical study provides best performance for decreasing the electric field in binary Tantala layers by integrating at least three nanolaminate sequences before the last high refractive index layer in the stack (Fig. 5 - right). The nanolaminate sequences are characterized by a well of $1 \mathrm{~nm}$ and a barrier of $20 \mathrm{~nm}$ resulting in an optical gap of $4.65 \mathrm{eV}$. The thin Tantala layers of $1 \mathrm{~nm}$ within the sequence are controlled in thickness during deposition applying time control. The electric field is dropped to $60 \%$ in the last Tantala layer.

Table 1. Comparision of the two calculated high relfective mirror designs applying Tantla as high refractive and Silica as low refractive index material.

\begin{tabular}{|c|c|c|}
\hline & Conventional HL stack & HL stack including nanolaminates \\
\hline Number of layers & 31 & 55 \\
\hline Total design thickness $[\boldsymbol{\mu m}]$ & 3.5 & 3.4 \\
\hline Spectral range $\Delta \boldsymbol{\lambda}[\mathbf{n m}]$ & $730-850$ & $770-830$ \\
\hline Reflectance within $\Delta \boldsymbol{\lambda}[\%]$ & $>99.9$ & $>99.9$ \\
\hline GDD within $\Delta \boldsymbol{\lambda}\left[\mathbf{f s}^{\mathbf{2}}\right]$ & 0 & 0 \\
\hline $\begin{array}{c}\text { AOI }\left[{ }^{\circ}\right] \\
{[\%]}\end{array}$ & 0 & 0 \\
\hline \begin{tabular}{c} 
Maximum electric field $\mathbf{T a}_{\mathbf{2}} \mathbf{O}_{\mathbf{5}}$ \\
\hline
\end{tabular} & 47 & 40 \\
\hline
\end{tabular}



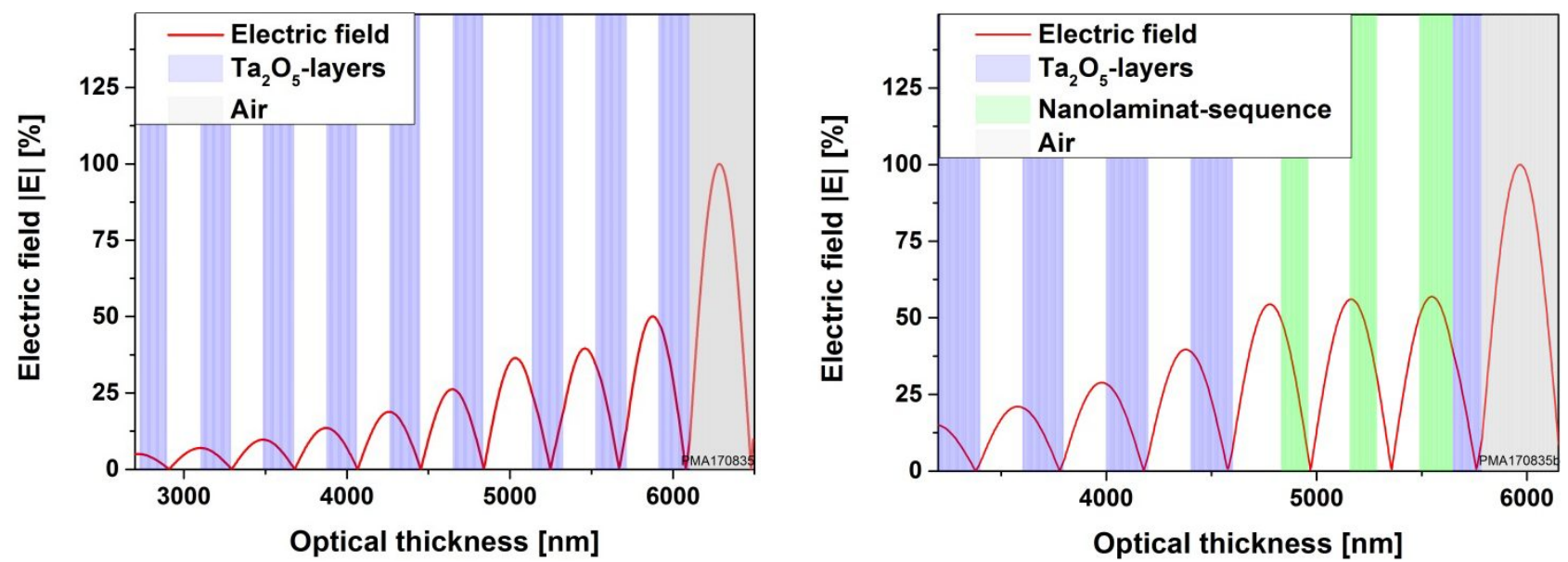

Figure 5. Electric field plotted against the optical thickness of a conventional $\mathrm{Ta}_{2} \mathrm{O}_{5}-\mathrm{SiO}_{2}$ (left) stack and a mirror containing nanolaminate sequences (right).

Both mirror designs are manufactured applying the described IBS process. Spectral transmittance measurements (Lambda 1050 - PerkinElmer) indicate no significant deviations from the expected spectra (Fig. 6 - lower row) within the target transmittance band. The respective transmittance data recorded after every layer are applied for a reverse engineering of the group delay dispersion band. Previous investigations revealed a good correlation between the measured group delay dispersion applying white light interferometry and the reverse engineering data [11 - 13]. The GDD of the conventional HR mirror is shifted in central wavelength with respect to the calculated design (Fig. 6 - upper row). Within $(760-860) \mathrm{nm}$ a GDD of $0 \pm 50 \mathrm{fs}^{2}$ is reached. The HR-mirror containing nanolaminate sequences depicts a good correlation of the GDD between the design and reverse engineering design. In the spectral range starting from 750 to $850 \mathrm{~nm}$, a GDD of $0 \pm 20 \mathrm{fs}^{2}$ can be expected.

In a next step, the laser induced damage thresholds of both mirrors were measured in a 10,000 on 1 procedure according to ISO 21254 [18]. The samples are irradiated close to normal incidence with laser pulses of a duration of $\tau_{\mathrm{P}}=150 \mathrm{fs}{ }^{2}$ at a repetition rate of $1 \mathrm{kHz}$. The measurement uncertainty for the retrieved LIDTvalues can be estimated with $10 \%$, because of the uncertainty of fluence values used for the tests The conventional HR mirror is characterized by an absolute LIDT of $\mathrm{H}_{\text {LIDT }}=0.71 \mathrm{~J} / \mathrm{cm}^{2}$. The mirror containing nanolaminate sequences offers a value of $0.82 \mathrm{~J} / \mathrm{cm}^{2}$. The LIDT could be increased by $16 \%$ by reducing the electric field in pure Tantala layers of around $7 \%$ with respect to a standard binary HR mirror.
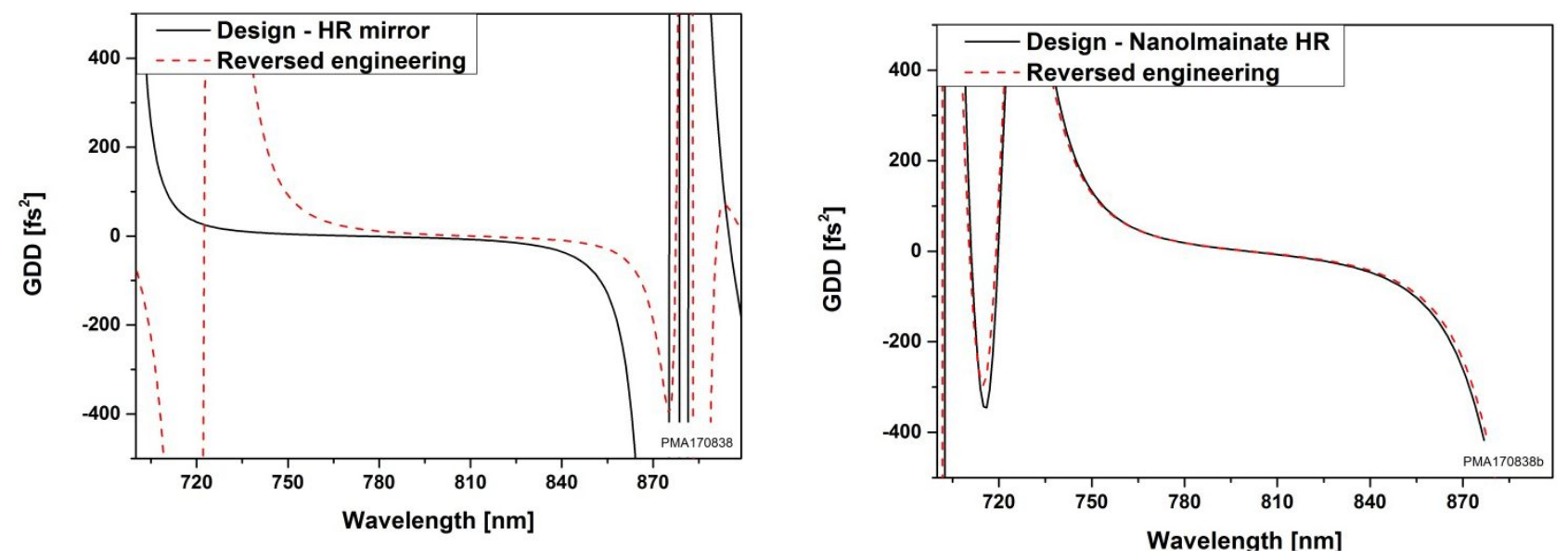

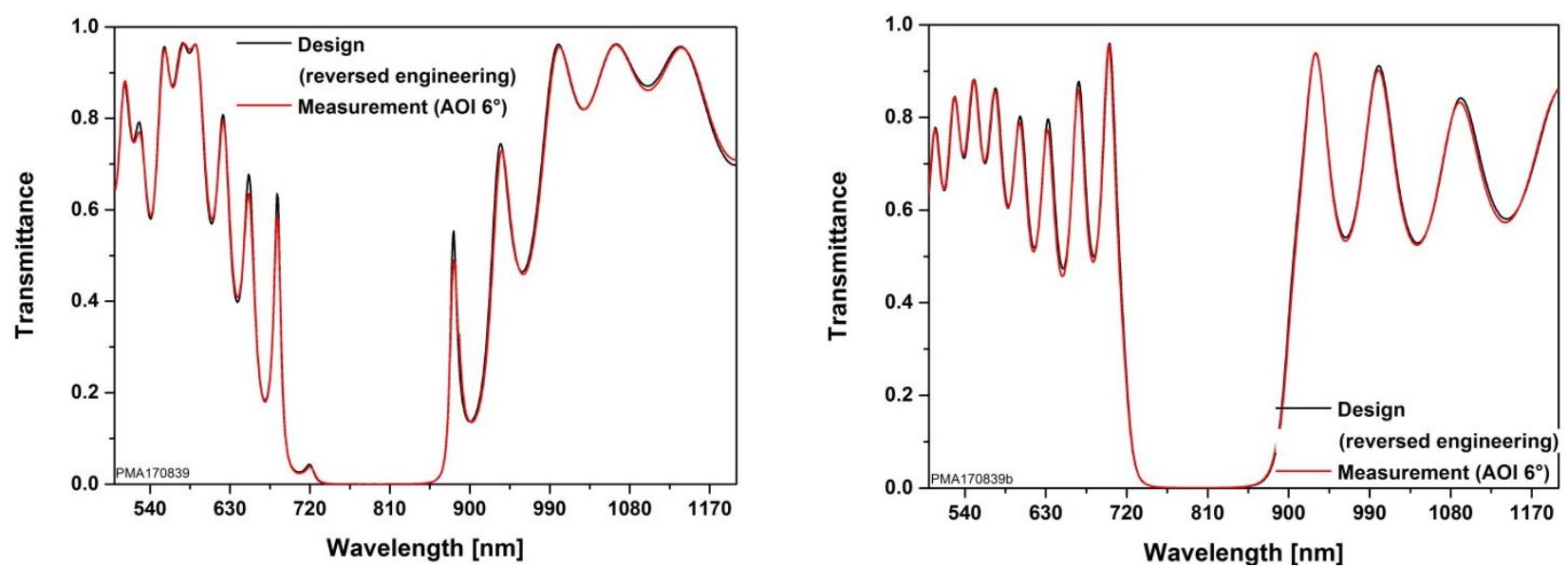

Figure 6. Designed group delay dispersion compared to a reverse engineering for the conventional HR mirror ( upper left) and mirror containing nanolaminate sequences (upper right). The lower row depicts the transmittance scan of the design and spectral measurement under an AOI of $6^{\circ}$ for the conventional HR (bottom left) and mirror containing nanolaminate sequences (bottom right)

The applied nanolaminate sequence is characterized by a 2.4 higher internal LIDT compared to binary $\mathrm{Ta}_{2} \mathrm{O}_{5}$. Thereby the internal LIDT is defined as $\mathrm{H}_{\text {int }}=\left|\mathrm{E}_{\max }\right| /\left|\mathrm{E}_{\text {inc }}\right| * \mathrm{H}$, with the relative ratio of the maximum electric field $\left|\mathrm{E}_{\max }\right|$ at a certain position in the stack and the incident wave $\left|\mathrm{E}_{\text {inc }}\right|$. The applied Software "Spektrum32" determines the ratio of the electric field according to the maximum possible value in vacuum, which is the top value in front of an HR-mirror with $100 \%$ [25]. The measured LIDT of the corresponding layer structure is depicted by H. In a multilayer stack, the LIDT can be expected to be determined by the layer with lowest internal LIDT [14]. In contrast to the complex behavior in chirped mirror, the weakest layer in a conventional HR mirror is surface layer. This refractive index layer in the stack has an electric field of $47 \%$ (fig. 5 - left). The internal LIDT of the mirror results to $H_{\text {int }}=0.33 \mathrm{~J} / \mathrm{cm}^{2}$. The maximum field strength inside the mirror containing nanolaminate sequences has a 1.22 times higher field strength compared to the binary Tantala layer in the HR stack. With respect to the RISED design by applying nanolaminate sequences, the most sensitive layer is the pure Tantala cover layer at the surface, which provides the possibility for further improvements. In detail, the damage of the mirror can be expected to be determined by the Tantala layer with an electric field of $40 \%$ (fig. 5 - right). The demand of a constant internal LIDT allows the approximation of the absolute LIDT of the high reflective mirror containing the nanolaminates to $\mathrm{H}_{\mathrm{cal}}=0.86 \mathrm{~J} / \mathrm{cm}^{2}$ [14]. The calculated value matches with the measurement result by a deviation of around $5 \%$. The design approach is expected to be very promising for complex designs like chirped mirrors with demanding specifications of the GDD. In such designs the electric field distribution is complex, and values are reached for high refractive index layers deep inside the layer stack. The substitution of such layers by nanolaminate sequences is expected to lead to a much higher improvement in LIDT, because the internal LIDT of applied nanolaminates is much higher with respect to binary high refractive index layers. In recently published studies a chirped mirror applying binary Tantala and Silica materials is calculated aiming a GDD of $-200 \mathrm{fs}^{2}$ and a spectral width of $100 \mathrm{~nm}$. Field intensities up to $390 \%$ occur in the $\mathrm{Ta}_{2} \mathrm{O}_{5}$ in the stack. Substituting this high refractive index layer by a nanolaminate structure the maximum field intensity in $\mathrm{Ta}_{2} \mathrm{O}_{5}$ could be dropped by a factor of 1.95 . The LIDT could be improved by almost $190 \%$ with respect to the binary chirped mirror [26]. In addition the application of dielectric nanolaminates offers the possibility to sputter single binary layers to tune the optical gap. The uniformity is expected to be more deterministic with respect to ternary composite layers, because the sputtering coil can be predicted more precisely for binary materials with compared to a changing composition of at least two target materials.

\section{Conclusion}

This paper reports about the application of dielectric amorphous nanolaminates. The optical gap can be tuned by embedding a high refractive index layer in a matrix of two low refractive index layers. By lowering the high refractive index layers below $4 \mathrm{~nm}$ the electron confinement starts to change. In good agreement with the finite potential well model the optical gap is increasing exponentially accompanied by a linear decreasing index of refraction. Furthermore, the optical gap remains constant for a fixed quantum well for varying thickness of the matrix layers, if the critical value for tunneling of electrons is not reached. The index of refraction can be increased exponentially for a constant optical gap by lowering the barriers. The displayed advantages are applied to increase the laser induced damage threshold of a conventional high reflective mirror for the near infrared spectral range. The applied nanolaminate sequence is 
characterized by a 2.4 higher internal LIDT with respect to a single $\mathrm{Ta}_{2} \mathrm{O}_{5}$ layer. An empirical design synthesis is performed to evaluate best performance of nanolaminate sequences in a high reflective mirror by reducing the maximum electric field in the pure high refractive index layers. The electric field could be reduced in binary Tantala layers by $30 \%$ with respect to a conventional binary $\mathrm{Ta}_{2} \mathrm{O}_{5} \mathrm{SiO}_{2}$ high reflective mirror. The LIDT of the mirror containing nanolaminate sequences can be approximated taking advantage of the determined internal LIDT of the binary high reflective mirror. The calculated and measured LIDT matches within the error bars and impose an improvement of the LIDT of almost $16 \%$. The design approach seems to be well suited for most complex designs with field intensities of several hundred percent in the high refractive index layer. The substitution of such layers by nanolaminate sequences with high internal LIDT values are expected to significantly improve the damage resistance of complex optical filters.

\section{Acknowledgements}

The authors thank LNQE for TEM and Volkswagen Stiftung (Hymnos) (ZN3061) and Deutsche Forschungsgemeinschaft (DFG) (Cluster of excellence 201 Quest) for the financial support.

\section{REFERENCES}

[1] Morgner, U., Kärtner, F. X., Cho, S. H., Chen, Y., Haus, H. A., Fujimoto, J. G, and Tschudi, T. ,"Sub-two-cycle pulses from a Kerr-lens mode-locked Ti: sapphire laser", Opt. Letters 24(6), 411-413, (1999).

[2] Brabec, T., Spielmann, C., Curley, P. F., and Krausz, F., "Kerr lens mode locking", Opt. Letters 17(18), 1292-1294, (1992).

[3] R.L.Forketal.,"Negative dispersion using pairs of prisms,"Opt. Letters, 9(5), 150-153, (1984).

[4] Lemoff, B., and Barty, C. P. J., "Cubic-phase-free dispersion compensation in solid-state ultrashort-pulse lasers", Opt. Letters, 18(1), 5759 (1993).

[5] Spielmann, C., Curley, P. F., Brabec, T., and Krausz, F., "Ultrabroadband femtosecond lasers," IEEE Journal of quantum electronics, 30(4), 1100-1114, (1994).

[6] R. Szipöcs, C. Spielmann, F. Krausz, and K. Ferencz, "Chirped multilayer coatings for broadband dispersion control in femtosecond lasers," Opt. Letters 19(3), 201-203 (1994).

[7] V. Pervak, O. Razskazovskaya, I. B. Angelov, K. L. Vodopyanov, and M. Trubetskov, "Dispersive mirror technology for ultrafast lasers in the range 220-4500 nm," Advanced Optical Technologies 3(1), 55-63 (2014).

[8] S. Rausch, T. Binhammer, A. Harth, J. Kim, R. Ell, F. X. Kärtner, and U. Morgner, "Controlled waveforms on the single-cycle scale from a femtosecond oscillator," Opt. Express 16(13), 9739-9745 (2008).

[9] R. Szipöcs, A. Stingl, C. Spielmann, and F. Krausz, "Pushing the limits of femtosecond technology: Chirped dielectric mirrors," Optics and Photonics News, 6(6), 16 (1995).

[10] D. Ristau, H. Ehlers, T. Gross, and M. Lappschies, “Optical broadband monitoring of conventional and ion processes,” Applied Optics, 45(7), 1495-1501 (2006)

[11] S. Schlichting, T. Willemsen, H. Ehlers, U. Morgner, and D. Ristau, "Fourier-transform spectral interferometry for in situ group delay dispersion monitoring of thin film coating processes," Opt. Express 24(20), 22516-22527 (2016).

[12] T. Willemsen, S. Schlichting, M. Gyamfi, M. Jupé, H. Ehlers, U. Morgner, and D. Ristau, "Improved LIDT values for dielectric dispersive compensating mirrors applying ternary composites," Proc. of SPIE 10014, 100141Z (2016).

[13] T. Willemsen, S. Schlichting, T. Kellermann, M. Jupé, H. Ehlers, U. Morgner, and D. Ristau, "Precise fabrication of ultra violet dielectric dispersion compensating mirrors," Proc. of SPIE 9627, 96271 U (2015).

[14] D. Ristau, Laser-Induced Damage in Optical Materials (CRC Press 2014).

[15] H. Kroemer, "Heterostructure bipolar transistors and integrated circuits," in Proceedings of the IEEE, (IEEE, 1982), pp. 13-25.

[16] T. Willemsen, M. Jupé, L. Gallais, D. Tetzlaff, and D. Ristau, "Tunable optical properties of amorphous Tantala layers in a quantizing structure," Opt. Letters 42(21), 4502-4505 (2017).

[17] T. Willemsen, P. Geerke, M. Jupé, L. Gallais, and D. Ristau, "Electronic quantization in dielectric nanolaminates," Proc. of SPIE 10014, $100140 \mathrm{C}(2016)$.

[18] ISO 21254, "Lasers and laser-related equipment - Test methods for laser-induced damage threshold," (2011).

[19] C. Kittel, Introduction to solid state (John Wiley \& Sons, 1966) Chap6.

[20] S. Schlichting, K. Heinrich, H. Ehlers., D. Ristau, "Online re-optimization as a powerful part of enhanced strategies in optical broadband monitoring," Proc. of SPIE 8168, 81681E (2011).

[21] B. Tatian, "Fitting refractive-index data with the Sellmeier dispersion formula," Appl. Optics 23(24), 4477-4485 (1984).

[22] J. Tauc, R. Grigorovici, and A. Vancu, "Optical properties and electronic structure of amorphous germanium," Physica status solidi 15(2), 627-637 (1966).

[23] M. Beaudoin, M. Meunier, and C. J. Arsenault, "Blueshift of the optical band gap: Implications for the quantum confinement effect in a-Si: H/a-SiN x: H multilayers," Physical Review B, 47(4), 2197 (1993).

[24] A.V. Tikhonravov, and M.K. Trubetskov, "OptiLayer software," http://www.optilayer.com 
[25] M. Dieckmann. Spektrum: software for optical interference coatings. Laser Zentrum Hannover e.V.,Hollerithallee 8, 30419 Hannover, Germany, 2002.

[26] T. Willemsen, M. Jupé, M. Gyamfi, S. Schlichting, and D. Ristau, "Enhancement of the damage resistance of ultra-fast optics by novel design approaches,” Optics express, 25(25), 31948-31959 (2017).

Proc. of SPIE Vol. 10447 1044712-8 